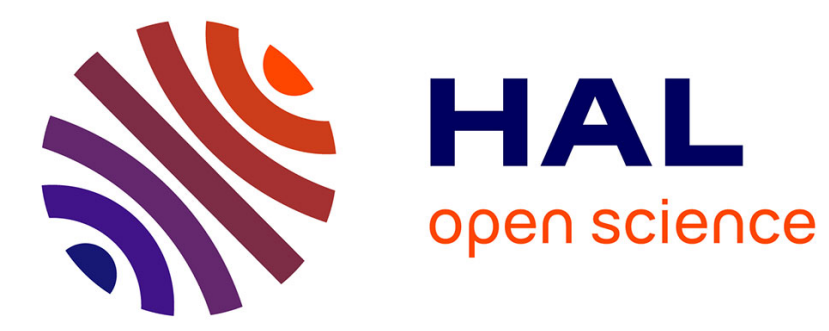

\title{
The effect of angular velocity and cycle on the dissipative properties of the knee during passive cyclic stretching: A matter of viscosity or solid friction
} Antoine Nordez, Peter Mcnair, Pascal Casari, Christophe Cornu

\section{- To cite this version:}

Antoine Nordez, Peter Mcnair, Pascal Casari, Christophe Cornu. The effect of angular velocity and cycle on the dissipative properties of the knee during passive cyclic stretching: A matter of viscosity or solid friction. Clinical Biomechanics, 2009, 24 (1), pp.77-81. 10.1016/j.clinbiomech.2008.10.004 . hal-01007360

\author{
HAL Id: hal-01007360 \\ https://hal.science/hal-01007360
}

Submitted on 22 Nov 2018

HAL is a multi-disciplinary open access archive for the deposit and dissemination of scientific research documents, whether they are published or not. The documents may come from teaching and research institutions in France or abroad, or from public or private research centers.
L'archive ouverte pluridisciplinaire HAL, est destinée au dépôt et à la diffusion de documents scientifiques de niveau recherche, publiés ou non, émanant des établissements d'enseignement et de recherche français ou étrangers, des laboratoires publics ou privés. 


\title{
The effect of angular velocity and cycle on the dissipative properties of the knee during passive cyclic stretching: A matter of viscosity or solid friction
}

\author{
A. Nordez ${ }^{\mathrm{a}, *}$, P.J. McNair ${ }^{\mathrm{b}}$, P. Casari ${ }^{\mathrm{c}}$, C. Cornu ${ }^{\mathrm{a}}$

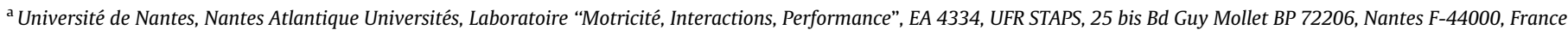

${ }^{\mathrm{b}}$ Health and Rehabilitation Research Centre, Auckland University of Technology, Private Bag 92006, Auckland 1, New Zealand

' Université de Nantes, Nantes Atlantique Universités, CNRS, Institut de recherche en Génie Civil et Mécanique, UMR 6183, UFR des Sciences et Techniques,
} \\ 2 rue de la Houssinière BP 92208, Nantes F-44000, France
}

\begin{abstract}
Background: The mechanisms behind changes in mechanical parameters following stretching are not understood clearly. This study assessed the effects of joint angular velocity on the immediate changes in passive musculo-articular properties induced by cyclic stretching allowing an appreciation of viscosity and friction, and their contribution to changes in torque that occur.

Methods: Ten healthy subjects performed five passive knee extension/flexion cycles on a Biodex dynamometer at five preset angular velocities $(5-120 \mathrm{deg} / \mathrm{s})$. The passive torque and knee angle were measured, and the potential elastic energy stored during the loading and the dissipation coefficient were calculated.

Findings: As the stretching velocity increased, so did stored elastic energy and the dissipation coefficient. The slope of the linear relationship between the dissipation coefficient and the angular velocity was unchanged across repetitions indicating that viscosity was unlikely to be affected. A difference in the $y$-intercept across repetitions 1 and 5 was indicative of a change in processes associated with solid friction. Electromyographical responses to stretching were low across all joint angular velocities.

Interpretation: Torque changes during cyclic motion may primarily involve solid friction which is more indicative of rearrangement/slipping of collagen fibers rather than the redistribution of fluid and its constituents within the muscle. The findings also suggest that it is better to stretch slowly initially to reduce the amount of energy absorption required by tissues, but thereafter higher stretching speeds can be undertaken.
\end{abstract}

\section{Introduction}

In humans, the passive mechanical properties of a musculoarticular complex (MAC), including structures spanning the joint (Riemann et al., 2001), can be determined using passive loading and unloading torque-angle responses (Gajdosik, 2001, 2004, 2005b; Magnusson, 1998; Magnusson et al., 1996, 1998; McNair et al., 2001, 2002; Nordez et al., 2006, 2008c). With such data many studies have shown that the passive torque and stiffness are altered immediately after cyclic (Magnusson et al., 1998; McNair et al., 2001, 2002; Nordez et al., 2008c) and static stretching protocols (Magnusson et al., 1996, 1998; McNair et al., 2001, 2002; Morse et al., 2008; Nordez et al., 2006, 2008c). While these passive stretching exercises are commonly performed in sports and rehabilitation, the mechanisms suggested for such changes are not well

\footnotetext{
* Corresponding author. Address: UFR STAPS, 25 bis bd Guy Mollet BP 72206 , Nantes F-44000, France.

E-mail address: antoine.nordez@univ-nantes.fr (A. Nordez).
}

understood and the immediate effects of stretching on the biomechanical properties of a MAC, such as the dissipative properties, remains a topic of continued interest to researchers.

The dissipative properties of a passive MAC can be evaluated by calculating a dissipation coefficient $(D C)$ from the normalized area of the hysteresis occurring during a loading/unloading cycle (Mandel, 1969; Nordez et al., 2008c; Persoz, 1960). It has been shown that the dissipative properties of the MAC are not modified after $6 \times 30$ s of static stretching, while they decreased significantly during cyclic stretching (Nordez et al., 2008c). These results suggest that the effects of stretching are qualitatively different after cyclic and static stretching and the mechanisms operating could be different.

In respect to dissipative properties, three mechanisms might explain changes in passive torque during cyclic stretching. Firstly, muscle fibers are known to exhibit a short range elastic component due to titin (Rassier et al., 2005) and/or a small proportion of attached cross-bridges (Proske and Morgan, 1999; Whitehead et al., 2001). However, a recent study has shown that ten short range and rapid stretches had no significant influence on the 
passive torque at the ankle joint (Morse et al., 2008). This result suggested that the short range elastic component is unlikely to be the mechanism leading to changes in passive mechanical properties during cyclic stretching. A second mechanism to be considered is that suggested by McNair et al. $(2001,2002)$ that changes in the structural arrangement of muscle could possibly occur during motion and induce muscle thixotropy. Thixotropy refers to a property of some materials showing a time-dependent change in fluid friction (i.e. viscosity). According to McNair et al. (2001, 2002), the more mobile constituents such as the polysaccharides and water might be redistributed during cyclic stretching. Finally Kubo et al. (2001) have shown that a static stretching protocol induces a decrease in tendon stiffness and that the hysteresis area of the tendon stress-strain relationship is decreased. Based upon the work of Stromberg and Wiederielm (1969), these authors proposed that collagen fibers could be rearranged/slip during stretching.

An in vivo technique for studying the contributions of tissues and fluids in the dissipative properties of the MAC has recently been developed (Nordez et al., 2008a). It involves the examination of passive torque-angle measures recorded at different joint angular velocities using an isokinetic dynamometer. From these measures, the dissipative properties of the MAC can be assessed across cycles of motion (Nordez et al., 2008c). Specifically, a linear model is fitted between the $D C$ across stretching angular velocities. Nordez et al. (2008a) established that the relationship between these variables is high with an $R^{2}$ of 0.99 . The linear relationship between the $D C$ and velocity allows an appreciation of solid friction and fluid friction (i.e. viscosity) in the system. The slope of the relationship is indicative of the viscous parameter while the intercept provides an appreciation of solid friction. The current work advances that of Nordez et al. (2008a) by examining the contribution of these parameters during cyclic stretching, the goal being to utilize this technique to determine and explain the effect of stretching velocity and cycle on the dissipative properties of the knee MAC. It was thought that such a project could contribute to a better understanding of the mechanisms related to the immediate effects of cyclic stretching.

\section{Methods}

\subsection{Subjects}

Ten healthy males (25.4 (SD 3.0) yr, height: $182.4($ SD 7.5$) \mathrm{cm}$, weight: 76.6 (SD 8.1) kg) volunteered to participate in this study and signed an informed consent form. These subjects participated in our previous study that established the techniques presented below. This study was conducted according to the Helsinki Statement (1964). Subjects practiced recreational sports, but did not participate in any strength or flexibility training at the time of the study. No subject had sustained a recent injury that may have affected the findings.

\subsection{Measurement techniques}

The experimental set-up has been previously described (Nordez et al., 2006, 2008c) and has been adapted from previous studies (Magnusson et al., 1996, 1998; Reid and McNair, 2004). Briefly, the Biodex system 3 research ${ }^{\circledR}$ isokinetic dynamometer (Biodex medical, Shirley, NY, USA) was used to measure torque produced in resistance to passive stretch $(T)$, knee joint angle $(\theta)$ and knee joint angular velocity $(\omega)$. Subjects were seated and the thigh was fastened using velcro straps to a thigh pad elevating it from horizontal. The trunk-thigh angle was adjusted to $60^{\circ}$, and the input axis of the dynamometer was aligned with the approximate axis of rotation of the knee joint. All subjects were unable to reach full knee extension in this position. All procedures began with the lower leg perpendicular to the thigh. This position was used to determine our reference knee angle expressed as $0^{\circ}$.

The maximal range of motion (RoM) was determined first (Nordez et al., 2006). The lower leg was passively extended ( $\omega=5 \mathrm{deg} /$ s), and the subjects used a stop switch when they perceived the maximum tolerable hamstring muscle stretch. This point was operationally defined as the maximal passive knee extension. The leg was then immediately returned to the starting position. The greatest angle change of three trials was considered as the maximal RoM. Subjects then performed a familiarization with the stretching protocol at the different tested velocities. Our previous study has shown that a five minute rest was sufficient to counteract the effects of five cyclic stretching repetitions (Nordez et al., 2008c). In the current study, a ten minute rest period was provided between the familiarization and the measurements. Thereafter, five passive loading/unloading cycles to $80 \%$ of the maximal RoM were performed at 5, 30, 60, 90 and $120 \mathrm{deg} / \mathrm{s}$ in a randomized order (Gajdosik et al., 2005a). Five minute rests were observed between each series at a given angular velocity. The foot was at rest in an equilibrium position during testing, and subjects were watched during the motion of the dynamometer. No movement of the foot was observed in any subjects.

Surface electromyographic signals (sEMG) of hamstring muscles were also recorded synchronously with the torque and angle data to ensure that no undesirable activation occurred during the stretching protocol. Using sEMG feedback, the subjects and the experimenter were able to visualize any activity in their muscles, and subjects were asked to stay as relaxed as possible. Bipolar electrodes (Delsys DE 02.3, Delsys Inc, Boston, USA) were placed on semitendinosus and biceps femoris muscles with a $10 \mathrm{~mm}$ interelectrode distance according to the recommendations of the surface electromyography for the Non-Invasive Assessment of Muscles project (SENIAM) (Hermens et al., 2000). In order to normalize the SEMG data recorded during the passive stretching trials, sEMG data were also collected during three maximal isometric knee flexions performed with the leg perpendicular to the thigh. $T, \theta, \omega$ and sEMG signals were sampled at $1000 \mathrm{~Hz}$ with an analog/ digital converter (Bagnoli 16, Delsys Inc, Boston, USA). All of the collected data were stored in a computer hard drive for further analysis.

\subsection{Data analyses}

The first and the fifth cycles were analyzed for all the velocities. Data were processed and calculated within Matlab ${ }^{\circledR}$ (The Mathworks, Natick, USA). Mechanical signals ( $T, \theta$ and $\omega$ ) were filtered using a Butterworth second order low pass dual filter $(10 \mathrm{~Hz})$. Recorded torque was then corrected for the limb mass and inertia. For that purpose, the torque due to the limb mass was recorded in the starting position (i.e. trunk-thigh angle: $60^{\circ}$, leg-thigh angle: $90^{\circ}$, the leg $30^{\circ}$ above horizontal) at the end of the protocol. In this position, it was assumed that there is negligible hamstring and quadriceps tension. Based on this limb mass estimation, the passive torque was corrected using the cosine function of the angle between the thigh and horizontal plane (Aagaard et al., 1995). The inertia of the Biodex level arm was determined during cycles performed at different velocities during an experiment with no subject on the dynamometer. The moment of inertia of the leg-foot was determined using regression equations (McFaull and Lamontagne, 1998) provided by Zatsiorski (1998). Using a sensitivity analysis, our previous results have shown that inertia estimation using predictive equations, and acceleration phase had negligible influence on our results (Nordez et al., 2008a).

At high velocities, a deceleration artefact was present, and a previous study (Nordez et al., 2008b) has shown, using the same 
methodology as in the present study, that the deceleration phase is less than $9^{\circ}$ at $120 \mathrm{deg} / \mathrm{s}$. Therefore, the initial and final $9^{\circ}$ were excluded from the range of motion used for all data analyses in order to avoid taking the deceleration artifact into account. Thereafter, for each preset velocity, the passive torque was determined every $2^{\circ}$ within the considered joint amplitude in order to obtain the three-dimensional relationship between passive torque, angle and the preset velocity. For all velocities, the energy stored during loading ( $E$, i.e. the area under the loading curve), the energy restituted ( $E R$, i.e. the area under the unloading curve) and the energy dissipated by the musculo-articular complex ( $E D$, i.e. the hysteresis area) were calculated. $E D$ was divided by $E$ to calculate the dissipation coefficient (DC, Eq. (1) (Nordez et al., 2008c).

$D C=E D / E=(E-E R) / E$

Relationships between $E, D C$ and the angular velocity were modeled using linear fits for each subject (Nordez et al., 2008a) in order to assess the $y$-intercept and the slope of these relationships.

sEMG data were processed according to the procedure described by Gajdosik et al. (2005a). The root mean square of sEMG signals (sEMG RMS) was calculated from the sEMG recorded while the subject's knee joint was being moved through its range of motion. sEMG RMS values were normalized to the maximal level recorded during the maximal isometric contractions. If sEMG RMS for a subject was greater than $1 \%$ of the maximum voluntary contraction during the stretching trials, then the subject's data were discarded (McNair et al., 2001, 2002).

\subsection{Statistical analysis}

After checking the normality of data distribution (KolmogorovSmirnov test), parametric statistical tests were performed using Statistica ${ }^{\circledR}$ software. $E$ and $D C$ calculated for the first and the fifth cycles (with all tested velocities pooled) were compared using a paired $T$-test. Changes in slopes and $y$-intercepts of linear relationships between $E, D C$ and angular velocity between the first and the fifth cycle were also analyzed using the paired T-test. Two $2 \times 2$ repeated measures (cycle $\times$ velocity) analysis of variances (ANOVAs) were performed to determine changes in $E$ and $D C$ between the first and the fifth cycle and between stretching at $5 \mathrm{deg} / \mathrm{s}$ and $120 \mathrm{deg} / \mathrm{s}$. Newman-Keuls post-hoc analyses were performed when appropriate. The critical level of significance in the present study was set at $P<0.05$.

\section{Results}

Three subjects were excluded from the study because their sEMG RMS levels during the passive stretching protocol were higher than $1 \%$ of the maximal level reached during isometric contractions. For the 1 st cycle, mean sEMG RMS were 0.06\% (SD 0.03\%), $0.11 \%$ (SD 0.06\%), $0.24 \%$ (SD 0.14\%), $0.30 \%$ (SD 0.20\%) and $0.46 \%$ (SD $0.21 \%$ ) of the maximal level reached during maximal isometric contractions at 5, 30, 60, 90 and $120 \mathrm{deg} / \mathrm{s}$, respectively. For the 5 th cycle, mean sEMG RMS were 0.05\% (SD 0.03\%), 0.08\% (SD 0.05\%), $0.33 \%$ (SD 0.24\%), $0.25 \%$ (SD 0.15\%) and 0.44\% (SD 0.22\%) at 5, 30, 60,90 and $120 \mathrm{deg} / \mathrm{s}$, respectively.

Between the first and the fifth cycle, $E$ decreased significantly $(P=0.000)$ by $16.3 \%$ (SD $4.9 \%$ ). Magnitudes of the decrease in $E$ after five cycles were similar at all angular velocities (Fig. 1, $-15.7 \%$ (SD 5.0\%), -16.1\% (SD 2.9\%), -16.3\% (SD 4.4\%), -16.6\% (SD 5.5\%), $-20.4 \%$ (SD 5.4\%) for angular velocities of 5, 30, 60, 90 and $120 \mathrm{deg} / \mathrm{s}$, respectively). Significant main effects were found for cycle $(P=0.000)$ and velocity $(P=0.000)$ were found. In addition a significant interaction $(P=0.033)$, indicating that $E$ de-

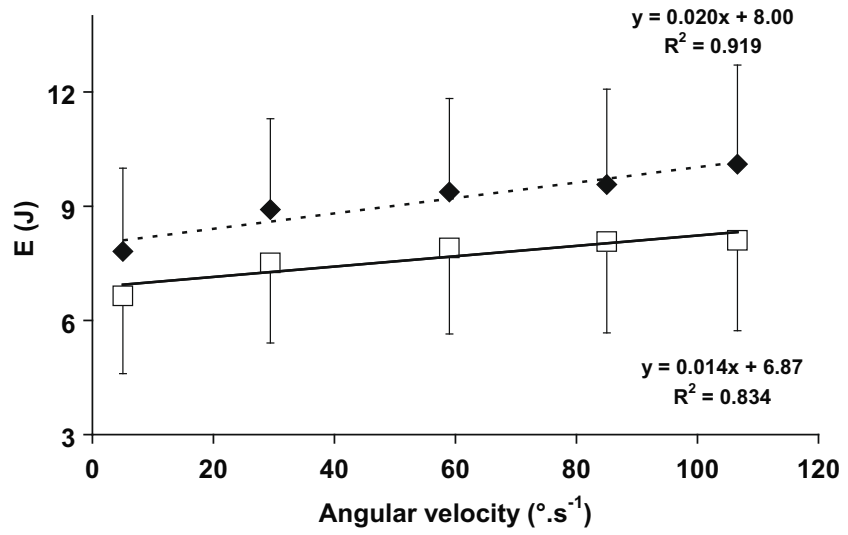

Fig. 1. Potential elastic energy stored during the loading $(E)$ as a function of the angular velocity for the first $(\checkmark)$ and the fifth $(\square)$ cycles.

Table 1

Slopes and $y$-intercepts of relationships between the potential elastic energy stored $(E)$, the dissipation coefficient $(D C)$ across stretching velocities for the first (1st) and the fifth (5th) cycles.

\begin{tabular}{|c|c|c|c|c|c|c|c|c|}
\hline \multirow[t]{3}{*}{ Subject } & \multicolumn{4}{|l|}{$E$} & \multicolumn{4}{|l|}{$D C$} \\
\hline & \multicolumn{2}{|c|}{$a\left(10^{-2} \mathrm{Js} / \mathrm{deg}\right)$} & \multicolumn{2}{|l|}{$b(\mathrm{~J})$} & \multicolumn{2}{|c|}{$a\left(10^{-3} \mathrm{~s} / \mathrm{deg}\right)$} & \multicolumn{2}{|l|}{$b$ (a.u.) } \\
\hline & $1 \mathrm{st}$ & 5 th & $1 \mathrm{st}$ & 5 th & $1 \mathrm{st}$ & 5 th & $1 \mathrm{st}$ & 5 th \\
\hline 1 & 2.71 & 2.28 & 8.55 & 6.95 & 0.76 & 0.63 & 0.47 & 0.41 \\
\hline 2 & 0.99 & 0.59 & 4.36 & 3.42 & 0.38 & 0.30 & 0.64 & 0.58 \\
\hline 3 & 3.47 & 1.48 & 6.92 & 6.31 & 0.24 & 0.16 & 0.31 & 0.30 \\
\hline 4 & 1.31 & 0.77 & 7.70 & 6.83 & 1.12 & 1.24 & 0.35 & 0.29 \\
\hline 5 & 1.88 & 1.62 & 9.98 & 8.44 & 1.27 & 1.34 & 0.44 & 0.39 \\
\hline 6 & 1.76 & 1.40 & 10.52 & 9.26 & 1.02 & 1.08 & 0.32 & 0.25 \\
\hline 7 & 1.96 & 2.02 & 8.21 & 7.06 & 0.43 & 0.52 & 0.50 & 0.44 \\
\hline Mean & 2.01 & 1.45 & 8.03 & 6.90 & 0.75 & 0.75 & 0.43 & 0.38 \\
\hline SD & 0.84 & 0.61 & 2.05 & 1.85 & 0.40 & 0.47 & 0.12 & 0.11 \\
\hline$P$ & 0.0327 & & 0.0001 & & 0.4223 & & 0.0001 & \\
\hline
\end{tabular}

Level of significance: $P<0.05$.

creased slightly but significantly more at $120 \mathrm{deg} / \mathrm{s}(-20.4 \%$ (SD $5.4 \%)$ ) in comparison to $5 \mathrm{deg} / \mathrm{s}(-15.7 \%$ (SD 5.0\%)). After five cycles of motion, the slope (cycle 1: $0.020 \mathrm{~J} \mathrm{~s} / \mathrm{deg}$ (SD 0.008); cycle 5: $0.015 \mathrm{~J} \mathrm{~s} / \mathrm{deg}(\mathrm{SD} 0.006) ; P=0.032$ ) and the $y$-intercept (cycle 1 : $8.03 \mathrm{~J}$ (SD 2.05); cycle 5: $6.90 \mathrm{~J}$ (SD 1.85); $P=0.000$ ) of the relationship between $E$ and the angular velocity decreased significantly (Table 1).

Between the first and the fifth cycle, the $D C$ decreased significantly $(P=0.000)$. Magnitudes of the decrease in $D C$ after five cycles were similar at all angular velocities (Fig. 2, $-12.1 \%$ (SD $5.4 \%$ ), $-14.1 \%$ (SD 3.4\%), $-11.7 \%$ (SD 3.5\%), -12.0\% (SD 4.5\%), $-12.3 \%$ (SD 2.4\%) for stretching angular velocities of $5,30,60,90$ and $120 \mathrm{deg} / \mathrm{s}$ respectively). Significant main effects were found for cycle $(P=0.000)$ and velocity $(P=0.007)$. The interaction between cycle $\times$ velocity was not significant $(P=0.243)$. After five cycles of motion, the slope of the relationship between the $D C$ and the angular velocity (cycle $1: 0.00075 \mathrm{~s} / \mathrm{deg}$ (SD 0.00040); cycle 5: $0.00075 \mathrm{~s} / \mathrm{deg}$ (SD 0.00047); $P=0.422$ ) was not significantly different while the $y$-intercept decreased significantly (cycle 1 : 0.432 (SD 0.117); cycle 5: 0.379 (SD 0.112); $P=0.000$ ) (Table 1).

\section{Discussion}

In order to attain a better understanding of the mechanisms responsible for the immediate effects of cyclic stretching, this study assessed the influence of joint angular velocity on changes in passive mechanical properties induced by cyclic stretching. A 


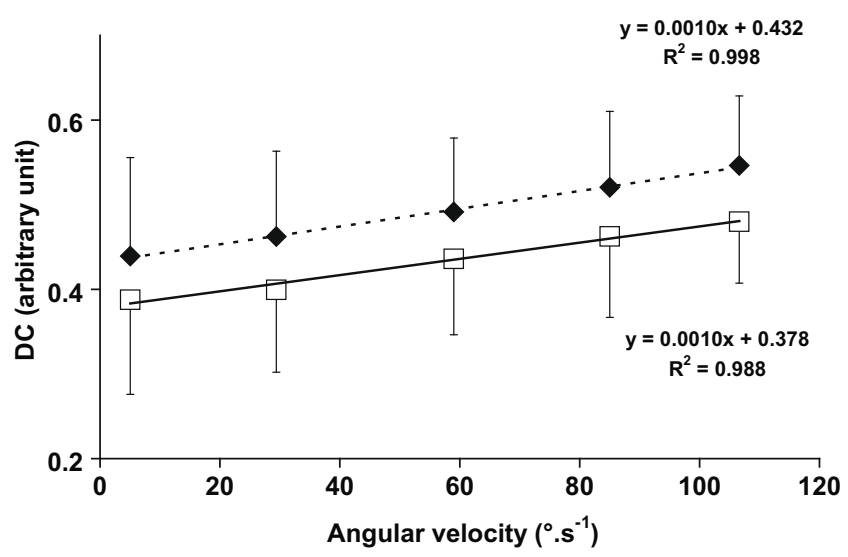

Fig. 2. Dissipation coefficient $(D C)$ as a function of the angular velocity for the first $(\diamond)$ and the fifth ( $\square$ ) cycles.

previous study examining $E$ and $D C$ and the effects of stretching considered a single joint angular velocity only (Nordez et al., 2008c). Building upon this work, our results show that $E$ and $D C$ increased with increases in the stretching velocity. The examination of the slope and intercept of the relationship between the $D C$ and joint angular velocity also allowed an appreciation of whether viscous damping and solid friction were notable mechanisms operating during passive cyclic stretching.

The findings showed that the slope of the relationship between $D C$ and the stretching velocity was not changed, while the $y$-intercept decreased significantly. Since the slope of the relationship between $D C$ and the stretching velocity is related to fluid friction (i.e. viscosity) while the $y$-intercept is related to solid friction (Nordez et al., 2008a), the observed changes are occurring primarily due to a decrease in solid friction. Thus McNair et al's $(2001,2002)$ proposal that decreases in viscosity occur mainly as a result of motion of polysaccharides and water in the tissues is unlikely to be so, at least for cyclic stretching activities. A mechanism involving solid friction is more likely, indicating that tissue changes contribute more to the decrease in passive torque during cyclic stretching. Considering the literature, a reorganization of muscle-tendon tissues could occur in two locations. Muscle fibers are known to exhibit a short range elastic component due to titin (Rassier et al., 2005) and/or a small proportion of attached cross-bridges (Proske and Morgan, 1999; Whitehead et al., 2001). However, as stated in the introduction, recent findings by (Morse et al., 2008) would suggest this last mechanism is less likely to be operating. Thus the findings of the current study are better explained by the suggestion that there is a rearrangement/slipping of collagen fibers (Kubo et al., 2001; Stromberg and Wiederielm, 1969).

While previous work has not assessed $E$ nor $D C$ across cycles of motion, McNair et al. (2002) have shown that related parameters (passive torque and stiffness) decreased more at $25 \mathrm{deg} / \mathrm{s}$ (torque: $-21 \%$, stiffness: $-31 \%$ for 120 s of cyclic stretching) compared to $5 \mathrm{deg} / \mathrm{s}$ (torque: $-13 \%$, stiffness: $-20 \%$ for 120 s of cyclic stretching). In contrast, in the present study changes in $E$ were not significantly different over the angular velocities that were similar to those of McNair et al (i.e. $-15.7 \%$ at $5^{\circ} \mathrm{deg} / \mathrm{s}$ compared to $-16.1 \%$ at $30 \mathrm{deg} / \mathrm{s}$ ). However, McNair et al. tested both angular velocities over a 2 min period and hence the number of motion cycles completed was quite different across their two angular velocities. The large changes observed by McNair et al. show how influential repeated cycles of motion are in inducing decreases in torque and stiffness.

EMG responses are thought to provide a protective mechanism to stop a muscle and/or joint from being damaged. Traditionally, authors (e.g. DeVries, 1962) have advocated the use of slow

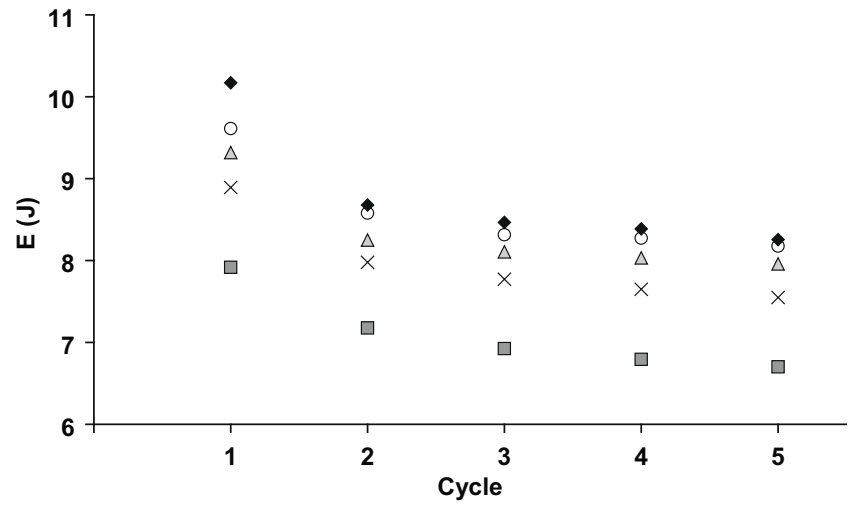

Fig. 3. Potential elastic energy stored during the loading $(E)$ for the five cyclic stretching repetitions at preset velocities of $5 \mathrm{deg} / \mathrm{s}(\boldsymbol{\square}), 30 \mathrm{deg} / \mathrm{s}(\times), 60 \mathrm{deg} / \mathrm{s}(\boldsymbol{\Lambda})$ $90 \mathrm{deg} / \mathrm{s}(\bigcirc), 120 \mathrm{deg} / \mathrm{s}(\bullet)$

stretches to minimize EMG responses and thus maximize the amount of stretch that can be applied to a joint's soft tissues. In the current study, the number of subjects who had EMG responses to the stretches was low, indicating that passive lengthening of tissues can be undertaken effectively in most individuals at joint angular velocities observed during gait activities. This finding was in agreement with previous works who noted minimal EMG activity in subjects up to a joint angular velocity of $120 \mathrm{deg} / \mathrm{s}$ (Gajdosik et al., 2005a; Kim et al., 2005). Similarly, (Lamontagne et al., 1997) noted minimal EMG responses in muscles at joint angular velocities up to $180 \mathrm{deg} / \mathrm{s}$, though these authors stretched the ankle joint in a range of motion that was not near maximum, and hence was less likely to generate the higher forces and strain in the soft tissues that would initiate reflex responses.

Although EMG responses may be low, our results also indicate that caution is needed in undertaking dynamic stretches. The finding that $E$ increased at higher joint angular velocities has relevance to injury of the tissues. Despite a lack of empirical evidence, for many years, it has been thought that ballistic stretches undertaken quickly might lead to injury. Our findings illustrate the concept that at higher speeds of stretch, a great amount of energy absorption capacity is required. If the muscle being stretched does not have that capacity then it will be damaged. Thus, our findings support the suggestion that cyclic stretching activities whether in preparation for vigorous sport or in rehabilitation scenarios where tissues are recovering from injury should be undertaken at slower speeds, at least initially, to decrease the amount of energy that must be absorbed. Related to these thoughts Fig. 3 shows the energy stored over cycles of motion, and indicates that within the first 2-3 cycles $E$ is reduced substantially, thus placing tissues at a lesser risk of injury. Therefore, these cycles are those that are best undertaken at a slow speed. Thereafter the speed of motion could be increased with less likelihood of injury.

\section{Summary and conclusions}

This study has provided new information related to the effect of stretching soft tissues dynamically. The findings provide evidence that fluid friction (i.e. the viscosity) is less likely to be playing a major role in torque changes observed during a cyclic stretching protocol. Therefore the motion of fluid constituents within the tissues is likely to be low during such motion. The significant decrease in solid friction indicates that tissues of the muscle-tendon unit are more likely to be involved and that the rearrangement/slipping of collagen fibers could be proposed to explain the observed changes. The low EMG responses to stretch provides support for 
the use of dynamic stretches at higher joint angular velocities, though a slower speed is recommended when performing the initial stretches to limit the amount of energy to be absorbed by the tissues.

\section{References}

Aagaard, P., Simonsen, E.B., Trolle, M., Bangsbo, J., Klausen, K., 1995. Isokinetic hamstring/quadriceps strength ratio: influence from joint angular velocity, gravity correction and contraction mode. Acta Physiol. Scand. 154, 421-427.

DeVries, H., 1962. Evaluation of static stretching procedures for improvement of flexibility. Res. Quart. Exerc. Sports 33, 222-229.

Gajdosik, R.L., 2001. Passive extensibility of skeletal muscle: review of the literature with clinical implications. Clin. Biomech. 16, 87-101.

Gajdosik, R.L., Vander Linden, D.W., McNair, P.J., Riggin, T.J., Albertson, J.S., Mattick, D.J., Wegley, J.C., 2004. Slow passive stretch and release characteristics of the calf muscles of older women with limited dorsiflexion range of motion. Clin. Biomech. 19, 398-406.

Gajdosik, R.L., Vander Linden, D.W., McNair, P.J., Riggin, T.J., Albertson, J.S., Mattick, D.J., Wegley, J.C., 2005a. Viscoelastic properties of short calf muscle-tendon units of older women: effects of slow and fast passive dorsiflexion stretches in vivo. Eur. J. Appl. Physiol. 95, 131-139.

Gajdosik, R.L., Vander Linden, D.W., McNair, P.J., Williams, A.K., Riggin, T.J., 2005b. Effects of an eight-week stretching program on the passive-elastic properties and function of the calf muscles of older women. Clin. Biomech. 20, 973-983.

Hermens, H.J., Freriks, B., Disselhorst-Klug, C., Rau, G., 2000. Development of recommendations for SEMG sensors and sensor placement procedures. Electromyogr. Kinesiol. 10, 361-374.

Kim, D.Y., Park, C.I., Chon, J.S., Ohn, S.H., Park, T.H., Bang, I.K., 2005. Biomechanical assessment with electromyography of post-stroke ankle plantar flexor spasticity. Yonsei. Med. J. 46, 546-554.

Kubo, K., Kanehisa, H., Kawakami, Y., Fukunaga, T., 2001. Influence of static stretching on viscoelastic properties of human tendon structures in vivo. J. Appl. Physiol. 90, 520-527.

Lamontagne, A., Malouin, F., Richards, C.L., 1997. Viscoelastic behavior of plantar flexor muscle-tendon unit at rest. J. Orthop. Sports Phys. Ther. 26, 244-252.

Magnusson, S.P., 1998. Passive properties of human skeletal muscle during stretch maneuvers a review. Scand. J. Med. Sci. Sports 8, 65-77.

Magnusson, S.P., Simonsen, E.B., Aagaard, P., Kjaer, M., 1996. Biomechanical responses to repeated stretches in human hamstring muscle in vivo. Am. J. Sports Med. 24, 622-628.
Magnusson, S.P., Aagard, P., Simonsen, E., Bojsen-Moller, F., 1998. A biomechanical evaluation of cyclic and static stretch in human skeletal muscle. Int. J. Sports Med. 19, 310-316.

Mandel, J., 1969. Aperçu sur les principaux comportements rhélogiques. In: Persoz, B. (Ed.), La rhéololgie. Masson \& Cie, Paris.

McFaull, S.R., Lamontagne, A., 1998. In vivo measurement of the passive viscoelastic properties of the human knee joint. Hum. Move. Sci. 17, 139-165.

McNair, P.J., Dombroski, E.W., Hewson, D.J., Stanley, S.N., 2001. Stretching at the ankle joint: viscoelastic responses to holds and continuous passive motion. Med. Sci. Sports Exerc. 33, 354-358.

McNair, P.J., Hewson, D.J., Dombroski, E., Stanley, S.N., 2002. Stiffness and passive peak force changes at the ankle joint: the effect of different joint angular velocities. Clin. Biomech. 17, 536-540.

Morse, C.I., Degens, H., Seynnes, O.R., Maganaris, C.N., Jones, D.A., 2008. The acute effect of stretching on the passive stiffness of the human gastrocnemius muscle tendon unit. J Physiol. 586, 97-106.

Nordez, A., Cornu, C., McNair, P., 2006. Acute effects of static stretching on passive stiffness of the hamstring muscles calculated using different mathematical models. Clin. Biomech. 21, 755-760.

Nordez, A., Casari, P., Cornu, C., 2008a. Effects of stretching velocity on passive resistance developed by the knee musculo-articular complex: contributions of frictional and viscoelastic behaviours. Eur. J. Appl. Physiol. 103, 243-250.

Nordez, A., Casari, P., Cornu, C., 2008b. Accuracy of Biodex system 3 pro isokinetic dynamometer in passive mode. Med. Eng. Phys. 30, 880-887.

Nordez, A., McNair, P.J., Casari, P., Cornu, C., 2008c. Acute changes in hamstrings musculo-articular dissipative properties induced by cyclic and static stretching. Int. J. Sports Med. 29, 414-418.

Persoz, B., 1960. Introduction à l'étude de la rhéologie. Dunod, Paris, France.

Proske, U., Morgan, D.L., 1999. Do cross-bridges contribute to the tension during stretch of passive muscle? J. Muscle Res. Cell. Motil. 20, 433-444.

Rassier, D.E., Lee, E.J., Herzog, W., 2005. Modulation of passive force in single skeletal muscle fibres. Biol. Lett. 1, 342-345.

Reid, D.A., McNair, P.J., 2004. Passive force, angle, and stiffness changes after stretching of hamstring muscles. Med. Sci. Sports Exerc. 36, 1944-1948.

Riemann, B.L., DeMont, R.G., Ryu, K., Lephart, S.M., 2001. The effects of sex, joint angle, and the gastrocnemius muscle on passive ankle joint complex stiffness. J. Athl. Train. 36, 369-375.

Stromberg, D.D., Wiederielm, C.A., 1969. Viscoelastic description of a collagenous tissue in simple elongation. J. Appl. Physiol. 26, 857-862.

Whitehead, N.P., Gregory, J.E., Morgan, D.L., Proske, U., 2001. Passive mechanical properties of the medial gastrocnemius muscle of the cat. J. Physiol. 536, 893903.

Zatsiorski, V., 1998. Kinematics of Human Motion, Champaign, IL. Human Kinetics. 